\title{
An improved quantitative measure of the tendency for volcanic ash plumes to form in water: implications for the deposition of marine ash beds
}

Christian T. Jacobs ${ }^{\mathrm{a}, \mathrm{b}, *}$, Tamara J. Goldin ${ }^{\mathrm{c}}$, Gareth S. Collins ${ }^{\mathrm{b}}$, Matthew D. Piggott ${ }^{\mathrm{b}, \mathrm{d}}$, Stephan C. Kramer ${ }^{\mathrm{a}, \mathrm{b}}$, H. Jay Melosh ${ }^{\mathrm{e}}$, Cian R. G. Wilson ${ }^{\mathrm{f}}$, Peter A. Allison ${ }^{b}$

${ }^{a}$ Institute of Shock Physics, Imperial College London, London SW7 2AZ, UK

${ }^{b}$ Department of Earth Science and Engineering, Imperial College London, London SW' 2AZ, UK

${ }^{c}$ Nature Geoscience, Nature Publishing Group, London N1 9XW, UK

${ }^{d}$ Grantham Institute for Climate Change, Imperial College London, London SWY $2 A Z$, $U K$

${ }^{e}$ Department of Earth, Atmospheric, and Planetary Sciences, Purdue University, Indiana 47907, USA

${ }^{f}$ Lamont-Doherty Earth Observatory, Columbia University, New York 10964, USA

\begin{abstract}
Laboratory experiments and numerical simulations have shown that volcanic ash particles immersed in water can either settle slowly and individually, or rapidly and collectively as particle-laden plumes. The ratio of timescales for individual and collective settling, in the form of analytical expressions, provides a dimensionless quantitative measure of the tendency for such plumes to grow and persist which has important implications for determining particle residence times and deposition rates. However, existing measures in the literature assume that collective settling obeys Stokes' law and is therefore controlled by the balance between gravitational forces and viscous drag, de-
\end{abstract}

\footnotetext{
*Corresponding Author

Email address: c.jacobs10@imperial.ac.uk (Christian T. Jacobs)
} 
spite plume development actually being controlled by the balance between gravitational forces and inertial drag even in the absence of turbulence during early times. This paper presents a new measure for plume onset which takes into account the inertial drag-controlled (rather than viscous drag-controlled) nature of plume growth and descent. A parameter study comprising a set of numerical simulations of small-scale volcanic ash particle settling experiments highlights the effectiveness of the new measure and, by comparison with an existing measure in the literature, also demonstrates that the timescale of collective settling is grossly under-estimated when assuming that plume development is slowed by viscous drag. Furthermore, the formulation of the new measure means that the tendency for plumes to form can be estimated from the thickness and concentration of the final deposit; the magnitude and duration of particle flux across the water's surface do not need to be known. The measure therefore permits the residence times of particles in a large body of water to be more accurately and practically determined, and allows the improved interpretation of layers of volcaniclastic material deposited at the seabed.

Keywords: Ash plumes, Settling rates, Volcaniclastic deposits, Computational modelling, Numerical simulations, Vertical density currents

\section{1. Introduction}

2 Explosive volcanism generates vast quantities of small ash particles which 3 can be transported over great distances, eventually depositing both on land 4 and on the seabed to form particle layers (Carey and Schneider, 2011). These 5 layers are a text-book example of isochroneity and have been used for strati- 
graphic correlation of past eruption events (e.g. Ver Straeten (2004, 2008)), allowing a wealth of information regarding their duration and frequency to be determined. Furthermore, ash deposits can potentially preserve information about the environmental conditions at the time of an event (Manville and Wilson, 2004). However, the process behind the settling of ash and the resulting formation of the particle layers is far from simple.

It was once assumed that the settling of ash in the deep sea occurred passively such that particles always descend slowly and individually under Stokes' law (Ledbetter and Sparks, 1979; Carey and Schneider, 2011), but several field-based observations have provided contradictory evidence. For example, following the 1991 eruption of Mount Pinatubo, ash fallout in the South China Sea settled at speeds of over $2 \mathrm{cms}^{-1}$ which is two to three orders of magnitude greater than the calculated Stokes' law velocities of individual particles (Wiesner et al., 1995). Through analogous laboratory experiments, Carey (1997) set out to explore this apparent contradiction in timescales and revealed the important role of vertical density currents in the rapid, collective transportation of material to the seabed.

The generation of vertical density currents is a complex multiphase process. Particles entering a body of water, either as fallout from ash clouds in the atmosphere or from a pyroclastic density current, undergo abrupt deceleration as they cross the air-water interface. Initially, slow and individual settling under Stokes' law ensues, allowing the particle concentration near the surface to rapidly increase and form a layer of particle-rich water over time. However, if the particle concentration in the layer is large enough for the particles to affect each other's settling through drag reduction and 
drifting such that the layer becomes gravitationally unstable, then finger-like Rayleigh-Taylor instabilities eventually form along the interface between the layer and the particle-free water below it. These instabilities grow exponentially to form plumes - clouds of particles that settle rapidly and collectively as vertical density currents.

Knowing whether plumes are likely to form, if at all, is important if one wishes to better determine the timescale of settling from the surface to the seabed. This can reveal information about the residence time of particles in the water and therefore the extent to which ambient ocean currents redistribute volcaniclastic material as it settles (Carey and Schneider, 2011). Similarly, knowing the rate of deposition can help determine the degree of bioturbation of the growing particle layer by marine organisms (Bramlette and Bradley, 1941). Plume formation also has implications for fossil preservation and stratigraphy. Rapid sedimentation has long been recognised as a means of increasing the likelihood that an organism could be preserved as a fossil (Seilacher et al., 1985) and so ash plume formation can impact upon the completeness of the fossil record. Perhaps one of the most celebrated and geologically significant examples of exceptional preservation beneath a marine ash deposit is that of the Neoproterozoic Ediacaran biota in Newfoundland which preserves some of the earliest metazoan fossils on Earth (Narbonne, 2005).

\subsection{Theoretical Considerations}

Quantitatively describing the tendency for plumes of particles to form in an ambient fluid has been achieved in previous works (Marsh, 1988; Goldin, 2008; Carazzo and Jellinek, 2012) through a dimensionless number $B$. This is 
defined in such a way that values of $B$ less than or equal to unity imply that plumes do not form, whereas a value greater than unity implies favourable conditions for plume growth and persistence. In particular, existing dimensionless numbers have been defined by the ratio of timescales for individual particle settling under Stokes' law and collective settling as a gravitationally unstable plume, such that

$$
B=\frac{\tau_{\text {individual }}}{\tau_{\text {collective }}} .
$$

That is, given information about the current state of Rayleigh-Taylor instabilities, the time required for particles to reach that state through individual and collective settling modes can be approximated using analytical expressions. Clearly a value of $B \gg 1$ implies favourable conditions for plume formation and persistence since collective settling happens over a shorter timescale (e.g. days or weeks in the ocean) than individual settling (e.g. months), whereas a value of $B \approx 1$ implies that plumes cannot form since the timescales of individual and collective settling are of the same order of magnitude. Note that a value of $B<1$ also implies that plumes cannot form, but when $B$ is defined by the ratio of timescales this value has no physical meaning except for the case of hindered settling (Kuenen, 1968) which is not considered here. The parameters needed to compute these expressions include the particle concentration and the thickness of the particle-rich layer which often have to be estimated in practice. Alternatively, the measure can be re-formulated in terms of a critical layer thickness that must be attained in order for pluming to take place (discussed later). This only requires knowledge of the mass influx across the water's surface and particle diameter 


$$
\tau_{\text {collective }}=\frac{18 \mu_{f}}{\alpha_{p}\left(\rho_{p}-\rho_{f}\right) g h},
$$

96 where $\alpha_{p}$ is the volume fraction of particles in the layer. Taking the ratio of 97 these two timescales yields the dimensionless number $B_{v v}$ :

$$
B_{v v}=\frac{\alpha_{p} h^{2}}{d_{p}^{2}} .
$$




\footnotetext{
${ }^{1}$ The Reynolds number is a dimensionless quantity defined as the ratio of inertial to viscous drag force.
} 
lidity of the measure for predicting the formation of plumes as particles settle in water is then evaluated and compared against $B_{v v}$. This is accomplished by (a) using data from the experiments by Carey (1997) which consider ash particles settling through a water tank, and (b) performing a parameter study through analogous numerical simulations with the multiphase computational fluid dynamics (CFD) code Fluidity (Piggott et al., 2008; Davies et al., 2011; Jacobs et al., 2013). The paper finishes with a discussion of the implications and applications of the new measure, other geophysical scenarios where the new measure could also be valid, and some concluding remarks. A list of notation used throughout the paper is provided in Appendix A.

\section{Derivation of the New Measure}

To derive a measure of the tendency of plumes to form which takes into account the fact that collective settling is slowed by inertial (rather than viscous) drag, consider the growth of wave-like instabilities with maximum amplitude $\delta$ at the interface between a particle-water layer of thickness $h$ and the particle-free water beneath it, as illustrated in Figure 1. The water is treated as an incompressible fluid, and the particles have an idealised spherical shape.

From Stokes' law, the timescale required for an individual spherical particle to settle through the layer of thickness $h$ is given by (2) previously. A timescale for the settling of a cloud of particles with a growing amplitude $\delta$ can be derived from an ordinary differential equation describing the latetime growth rate of Rayleigh-Taylor instabilities (Ristorcelli and Clark, 2004; Youngs, 1984), 


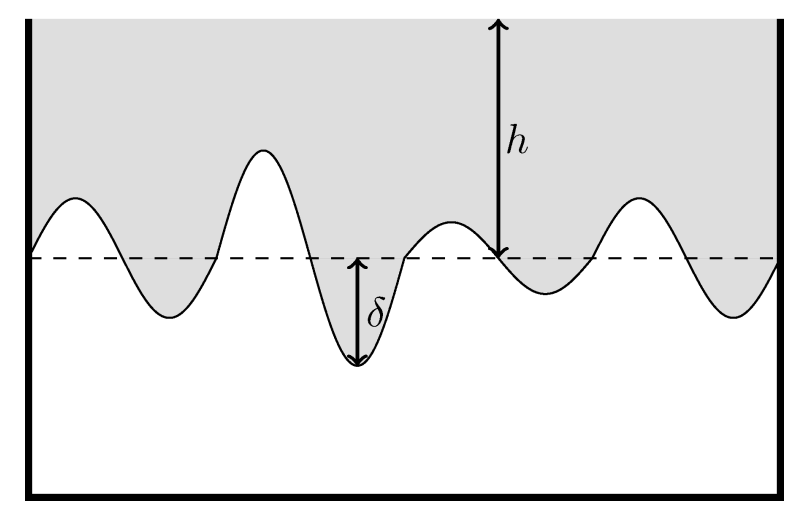

Figure 1: Illustration of particle plumes forming in a tank of water. The height of the particle-rich layer is denoted by $h$. The amplitude of the longest growing instability is denoted by $\delta$.

$$
\frac{d \delta}{d t}=2 \sqrt{\beta \operatorname{Atg} \delta}
$$

where $\beta$ is a dimensionless constant growth parameter, At $=\frac{\rho-\rho_{f}}{\rho+\rho_{f}}$ is the Atwood number, and $\rho$ is the bulk density of the plume defined as $\rho=$ $\alpha_{f} \rho_{f}+\alpha_{p} \rho_{p}$ where $\alpha_{f}$ is the volume fraction of the fluid. In this work, $\beta=0.03$ which is within the range of values estimated by experimental and numerical techniques (Dimonte and Schneider, 2000; Dimonte et al., 2004). This expression can be readily integrated to provide an expression for $\tau_{\text {collective }}$, given by (Youngs, 1984)

$$
\tau_{\text {collective }}=\sqrt{\frac{\delta}{\beta \mathrm{Atg}}} .
$$

Note that the initial condition $\delta(t=0)=0$ has been applied here. Although $t=0$ is supposed to be the point at which the flow reaches self-similarity 
(that is, when the flow behaviour appears the same on any scale) such that the initial condition becomes $\delta(t=0)=\delta_{0}$ for some $\delta_{0}>0$, this work chooses $t=0$ to correspond to the very start of the numerical simulation. This choice was shown a posteriori to still provide a consistently close approximation to the growth rate of the plumes across all simulations, even during very early times. Furthermore, this choice was made in order to be consistent with the expression for $\tau_{\text {individual }}$ and to avoid any ambiguity in deciding exactly when the flow becomes self-similar.

Taking the ratio of (2) and (6) yields the new dimensionless number

$$
B_{v i}=\frac{18 h \mu_{f}}{d_{p}^{2}} \sqrt{\frac{\alpha_{p} \beta}{\left(\rho+\rho_{f}\right)\left(\rho_{p}-\rho_{f}\right) \delta g}} .
$$

It should be emphasised that this dimensionless quantity assumes that the ambient fluid is incompressible, and that individual particle settling is controlled by the balance between gravitational forces and viscous drag, whereas plume growth and descent (i.e. collective particle settling) is controlled by the balance between gravitational forces and inertial drag. Additional measures can be derived for a compressible ambient fluid (Goldin, 2008), which is important for scenarios in which particles with a high initial momentum move through the atmosphere, and for different regimes of individual and collective particle settling. For completeness, the $B_{i i}$ measure appropriate for very coarse-grained particles that settle individually at Reynolds numbers much greater than unity, implying that the inertial drag force dominates viscous drag, is presented in Appendix B. However, this measure is not tested here. 


\section{Numerical Simulations}

To determine the ability of $B_{v i}$ and $B_{v v}$ to predict plume onset, a suite of two-phase numerical simulations of particle settling in water was performed using a multiphase computational fluid dynamics code called Fluidity, varying the particle diameter and constant particle mass flux (into the water from above) over a range that encompassed the laboratory particle settling experiments of Carey (1997). The size of the water tank in the simulations was $0.3 \mathrm{~m} \times 0.3 \mathrm{~m} \times 0.7 \mathrm{~m}$, replicating the geometry of Carey's experiments.

Initially, no particles were present in the domain, except along the surface where random perturbations in the particle volume fraction were introduced such that $10^{-7} \leq \alpha_{p} \leq 10^{-5}$. This essentially 'seeded' instabilities in the growing particle-water layer so that plumes could form. For numerical reasons, $\alpha_{p}$ was bounded below by a value of $10^{-7}$ instead of zero to avoid singularities in the system of linear equations. The velocity of both phases, denoted $\mathbf{u}_{f}$ and $\mathbf{u}_{p}$ respectively, was set to $\mathbf{0} \mathrm{ms}^{-1}$ (where $\mathbf{0}$ is the zero vector) at $t=0 \mathrm{~s}$. Throughout the simulations, no-normal flow conditions $\mathbf{u}_{f} \cdot \mathbf{n}=0$ and $\mathbf{u}_{p} \cdot \mathbf{n}=0$ (where $\mathbf{n}$ is the normal vector) were enforced along each boundary of the domain to prevent the fluid and particles from exiting. Particles entered the domain through the top boundary at a constant user-specified mass flux rate (defined later).

The following physical parameters were used and remained constant throughout all simulations: $\rho_{p}=2,340 \mathrm{kgm}^{-3}, \rho_{f}=1,000 \mathrm{kgm}^{-3}, \mu_{f}=0.001$ Pas, and $g=9.8 \mathrm{~ms}^{-2}$. The particle phase was assumed to be inviscid such that $\mu_{p}=0$ Pas. The range of mass flux was $2.50 \times 10^{-4}-6.11 \times 10^{-4} \mathrm{kgm}^{-2} \mathrm{~s}^{-1}$ (the range determined for the eruption of Mount St Helens on 18 May 1980 
(Sarna-Wojcicki et al., 1981; Scheidegger et al., 1982; Carey, 1997)), and $d_{p}$ ranged between 20 and $64 \mu \mathrm{m}$ as per the experiments by Carey (1997). In total, four different mass fluxes and six different particle diameters within these ranges were chosen, detailed in Table 1.

The domain was discretised using an unstructured mesh of solution nodes, composed of triangular and tetrahedral elements in two and three dimensions respectively, produced by Gmsh (Geuzaine and Remacle, 2009). The characteristic element length was fixed at $0.0025 \mathrm{~m}$, except in the preliminary three-dimensional simulation mentioned in the next paragraph which used mesh adaptivity (Piggott et al., 2008) to optimise the mesh throughout the simulation and place high resolution only where necessary in order to reduce computational costs; in this case, the upper and lower bounds on the element length were set to $0.1 \mathrm{~m}$ and $10^{-5} \mathrm{~m}$ respectively (Jacobs, 2013). The spatial discretisation of the model equations was performed using a Galerkin finite element method for the continuity and momentum equations, and a control volume method for the volume fraction fields (Jacobs et al., 2013; Jacobs, 2013). The implicit backward Euler method was used for the temporal discretisation, in conjunction with an adaptive time-stepping scheme which maximised the time-step subject to a Courant number of 0.5. All simulations were performed until $t=600 \mathrm{~s}$, which was enough time for plumes to form for all combinations of particle diameters and mass fluxes.

To establish any possible effect of problem geometry on plume formation, both 2D and 3D simulations were first performed using $d_{p}=48 \mu \mathrm{m}$ and a mass flux of $4.72 \times 10^{-4} \mathrm{kgm}^{-2} \mathrm{~s}^{-1}$ (see Figure 2). In both cases, initial particle settling happened individually at the appropriate Stokes' law veloc- 


\begin{tabular}{ccc}
\hline Reference & Mass flux $\left(\mathrm{kgm}^{-2} \mathrm{~s}^{-1}\right)$ & $d_{p}(\mu \mathrm{m})$ \\
\hline A1 - A6 & $2.50 \times 10^{-4}$ & $26,32,40,48,56,64$ \\
B1 - B6 & $3.61 \times 10^{-4}$ & $26,32,40,48,56,64$ \\
C1 - C6 & $4.72 \times 10^{-4}$ & $26,32,40,48,56,64$ \\
D1 - D6 & $6.11 \times 10^{-4}$ & $26,32,40,48,56,64$ \\
E1 - E2 & $4.72 \times 10^{-4}$ & 26,48 \\
\hline
\end{tabular}

Table 1: Reference table for the 24 simulations in the numerical parameter study (A1 A6, B1 - B6, C1 - C6 and D1 - D6), and for the experimental data points (E1 - E2).

ity, forming a uniform layer of thickness $h$. Eventually, instabilities at the base of this layer grew into plumes that settled to the base of the tank much more rapidly than the initial, individual particle settling speed. The layer thickness, particle volume fraction and time at the onset of plume formation differed by less than $10 \%$ between the $2 \mathrm{D}$ and 3D simulations. Therefore, for computational expedience, only 2D simulations were performed for the remaining particle diameters and mass fluxes. Note that for some simulations the nominal $0.3 \mathrm{~m} \times 0.7 \mathrm{~m}$ domain was extended in the vertical direction to accommodate plumes that grew longer than $0.7 \mathrm{~m}$.

To quantify the conditions at the onset of plume formation and hence evaluate the accuracy of the dimensionless quantities for predicting plume onset ( $B$ values), the values of $h$ and $\delta$ needed to be extracted from the simulation results. By assuming that particles in the layer settle under Stokes' law (at least until plumes have formed), the layer thickness $h$ was consistently found using the Stokes' law settling velocity multiplied by the time at the onset of pluming. This assumption was tested a posteriori and shown to be 


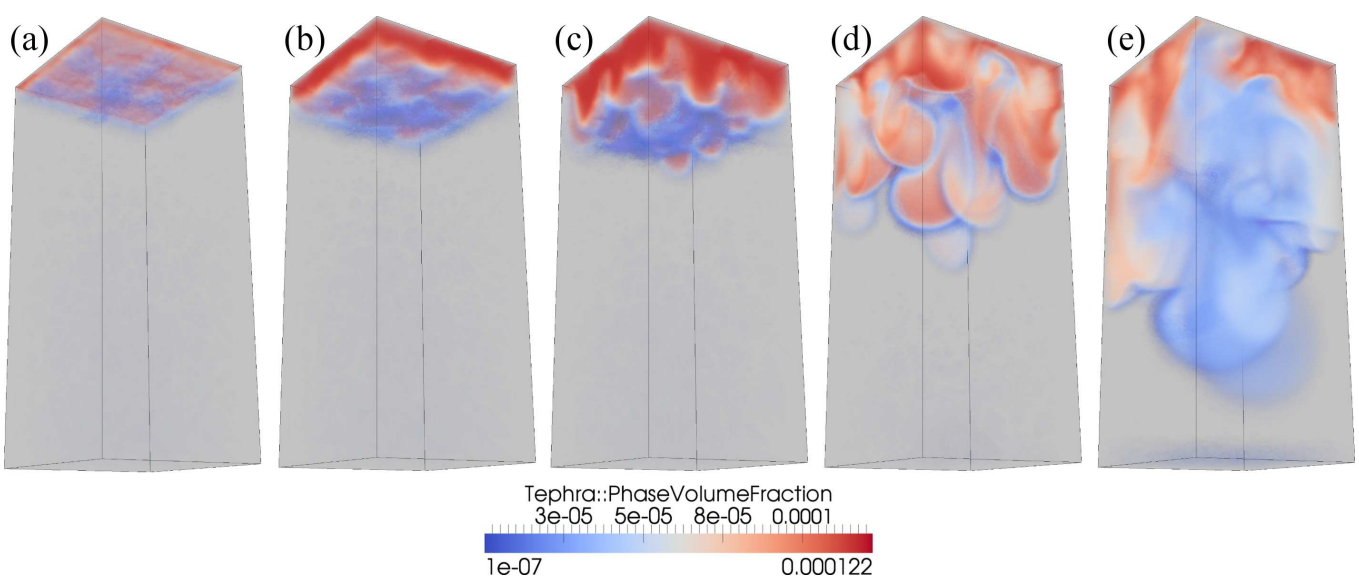

Figure 2: Three-dimensional simulation of particles settling through a tank of water at $t=($ a) 10, (b) 30, (c) 50, (d) 80, and (e) $120 \mathrm{~s}$. All visualisations show the whole $0.3 \mathrm{~m}$ $\times 0.3 \mathrm{~m} \times 0.7 \mathrm{~m}$ domain.

valid across all simulations. At a given time, the head of the growing plume of greatest amplitude $\delta$ was calculated by finding the lowermost position of the $10^{-5}$ particle volume fraction contour. This contour was chosen a posteriori as a sensible lower bound on the volume fraction of particles in the layer. The amplitude $\delta$ was then computed by taking the difference between the depth of the layer and the position of the plume head.

As one might expect, there is a certain amount of ambiguity involved when deciding when an instability is developed enough to be defined as a plume. Since the amplitude of a growing instability is known to be a function of the layer thickness (Manville and Wilson, 2004), this work defined the onset of pluming as the moment when $\delta=h$. The validity of this choice is discussed in Section 5. At this time, the quantities $h$ and $\alpha_{p}$ were determined, and the dimensionless numbers $B_{v v}$ and $B_{v i}$ were calculated. 


\section{Experimental Data}

The experiments performed by Carey (1997) used ultrasound imaging to track particle positions, which did not permit the accurate measurement of the parameters $h$ and $\alpha_{p}$. Some assumptions were therefore made in order to calculate estimates for experimental values of $B_{v v}$ and $B_{v i}$ for comparison with the numerical simulations. Assuming that particles in the growing particle-laden layer settled at Stokes' law velocity, $\mathbf{u}_{p}=\mathbf{u}_{\text {stokes }}$, the distance the particles had travelled at the time of plume onset, $t_{\text {onset }}$, provided an approximation for the layer thickness:

$$
h \approx\left|\mathbf{u}_{\text {stokes }}\right| t_{\text {onset }} .
$$

Furthermore, assuming the volume fraction of particles in the layer was uniform (because of the constant mass flux), and the total volume of the layer (including the water) was given by

$$
V_{\text {layer }}=h A \text {, }
$$

where $A$ is the area through which particles fluxed in $\left(A=0.9 \mathrm{~m}^{2}\right.$ for these particular experiments), then

$$
\alpha_{p}=\frac{V_{p}}{V_{\text {layer }}},
$$

where $V_{p}$ is the volume occupied by the particles. The mass flux of particles per unit area, $\dot{M}_{p}$, was used to calculate the volumetric flux per unit area $\dot{V}_{p}$ using 


$$
\dot{V}_{p}=\frac{\dot{M}_{p}}{\rho_{p}} .
$$

From this, the volume of the particle phase in the layer was calculated as

$$
V_{p}=A \dot{V}_{p} t_{\text {onset }},
$$

and the volume fraction followed from

$$
\alpha_{p}=\frac{V_{p}}{V_{\text {layer }}} .
$$

Carey (1997) noted that plumes had formed after approximately $30 \mathrm{~s}$ in experiment 96-5 which used 20-32 $\mu$ m diameter particles, and after approximately $60 \mathrm{~s}$ in experiment $96-1$ which used $32-64 \mu \mathrm{m}$ diameter particles. These times were used as approximations to $t_{\text {onset }}$ for the purpose of estimating $B_{v v}$ and $B_{v i}$, giving two data points for each measure, denoted E1 and E2 (see Table 1).

\section{Evaluation of the Measures}

The results from the parameter study reinforced the expected relationship between the particle diameter, mass flux and layer instability. Smaller particle sizes decrease the time required for plume onset because the slower Stokes' law settling results in a higher average particle concentration in the near-surface layer. This behaviour was also witnessed in the experiments performed by Carey (1997) where, for two ranges of particle diameter (20$32 \mu \mathrm{m}$ and $32-64 \mu \mathrm{m}$ ), there was a difference of approximately $30 \mathrm{~s}$ in the onset time. Similarly, a higher particle flux also causes a denser build-up of particles in the growing layer, further encouraging plume formation. 
As expected, the calculated values of $B_{v v}$ and $B_{v i}$, shown in Figures 3a and $3 \mathrm{~b}$ respectively, are all greater than unity since the parameters $h$ and $\alpha_{p}$ were measured at the point where plumes formed. Most importantly, the values from the measure $B_{v i}$ (which assumes that collective settling is slowed by inertial drag) lay consistently on a particular contour $(\sim 1.2)$, whereas the values from the measure $B_{v v}$ (which assumes that collective settling obeys Stokes' law and is therefore slowed by viscous drag) did not. In theory, one would expect plume onset to occur at a constant $B$ value because the definition of when a plume has formed does not change between simulations. By correctly describing the drag on the plumes, the $B_{v i}$ measure robustly estimated the timescale of collective particle settling, even when the system became more and more unstable and non-linear as a result of increasing particle diameter and flux rate. In contrast, the $B_{v v}$ measure grossly underestimated the timescale of collective settling.

Plume formation in every numerical simulation was robustly predicted by a $B_{v i}$ value of $\approx 1.2$. This threshold value for $B_{v i}$ was derived by defining $\delta=h$ as the condition for the onset of pluming. While the coefficient of $h$ in this expression was chosen arbitrarily, other coefficients close to unity would still result in a consistent plume-onset $B_{v i}$ value, but the exact threshold value would differ from 1.2. This is because for any $\delta$ proportional to $h$ the ratio of timescales between individual and collective particle settling is the same to within a constant factor for a given plume scenario.

Although the estimated experimental data points do not follow an exact contour for either measure, the two experimental $B_{v i}$ values are much more consistent than the two $B_{v v}$ values. The small discrepancy in the $B_{v i}$ values 

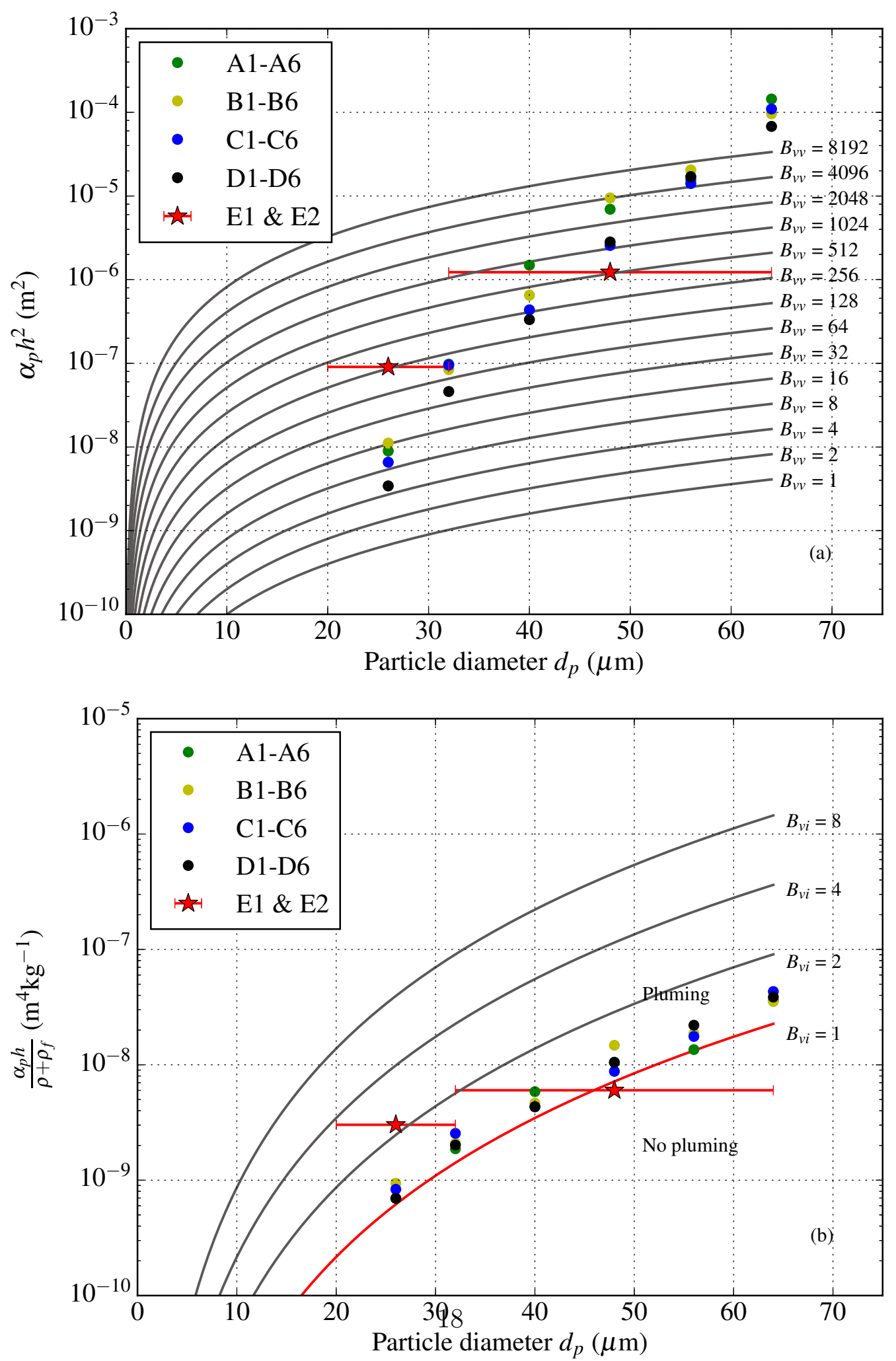

Figure 3: $B_{v v}$ and $B_{v i}$ results (presented in (a) and (b), respectively) using $\delta=h$. Several contours of $B_{v v}$ and $B_{v i}$ are given by solid lines. Due to the differences in the formulations of the measures, different quantities were considered along the $y$-axis. These quantities are related to the input mass flux of particles across the water surface, and are functions of the volume fraction of particles $\alpha_{p}$, the layer thickness $h$, the fluid density $\rho_{f}$ and the bulk density $\rho$. 
is easily explained by the ambiguity in plume onset time, which could not be accurately determined from the ultrasound images. Moreover, the approximate time of plume onset in the experiments does not necessarily correspond to the point at which $\delta=h$, thus potentially introducing further uncertainty in the experimental estimates.

At earlier times when plumes had not formed (i.e. before the point at which $\delta=h$ ), the values of $B_{v v}$ and $B_{v i}$ were also calculated to show that $B_{v i}$ is less than unity, while $B_{v v}$ is much greater than unity, demonstrating the inaccuracy of the measure that assumes collective settling is slowed by viscous drag. Simulation C4 is considered here for demonstration purposes because the relatively low mass flux and large particle diameter favoured the stability of the growing layer. Figure $2 \mathrm{a}$ shows the particle volume fraction at $t=10 \mathrm{~s}$. Clearly plumes had not formed at this point, and only very small initial perturbations (with $\delta \ll h$ ) are present along the base of the layer. It was found that all particles were still travelling at their Stokes' law velocity at this point in time. The $B_{v i}$ measure yielded a value less than unity $(\sim 0.3)$, correctly implying that individual particle settling dominated the dynamics. This also agrees with an estimated $B_{v i}$ value of $\sim 0.37$ (see the contour plot in Figure 4), computed using estimates for the volume fraction and layer thickness as described in Section 4. However, a $B_{v v}$ value of $\sim 15$ implied that plumes were already well into the growth stage. This demonstrates that the measure which assumes collective settling is slowed by viscous drag grossly under-estimates the timescale of plume growth and descent. On the other hand, the new measure which assumes collective settling is slowed by inertial drag is able to more accurately measure the tendency for plumes to 
form at early times.

\subsection{Alternative Formulation}

The measures in their current form require knowledge about the state of the layer, in particular the layer thickness, the volume fraction of particles within it, and (in the case of $B_{v i}$ ) the amplitude of the growing instabilities. Given this information, the non-dimensional number can be used to determine whether plumes will form. These quantities have to be estimated in practice since measuring them after or during an eruption event would be infeasible or impossible. However, as an alternative to calculating $B_{v v}$ and $B_{v i}$ directly from the state of the system, the measures can be re-formulated in terms of a critical layer thickness, denoted $h_{\text {crit }}$. For pluming to occur, the value of $h$ must satisfy $h_{\text {crit }}<h<H$, where $H$ is the height of the water column. The thickness of the layer $h$ can be estimated throughout time using Stokes' law since the particles within the layer settle individually. Furthermore, the critical value is expressed only in terms of the volumetric influx of particles and the particle diameter, such that the measures can be useful regardless of whether the exact values for $h, \delta$ and $\alpha_{p}$ are known.

By using a similar technique to that used when estimating the values of $B_{v v}$ and $B_{v i}$ from the experiments of Carey (1997), an expression for $\alpha_{p}$ (assumed to be constant and uniform in the layer) was formulated:

$$
\alpha_{p}=\frac{\dot{V}_{p}}{\left|\mathbf{u}_{\text {stokes }}\right|},
$$

where $\dot{V}_{p}$ is the volumetric flux (per unit area) and $\mathbf{u}_{\text {stokes }}$ is the Stokes' law velocity. This was then used to re-arrange both measures in terms of $h$, and 


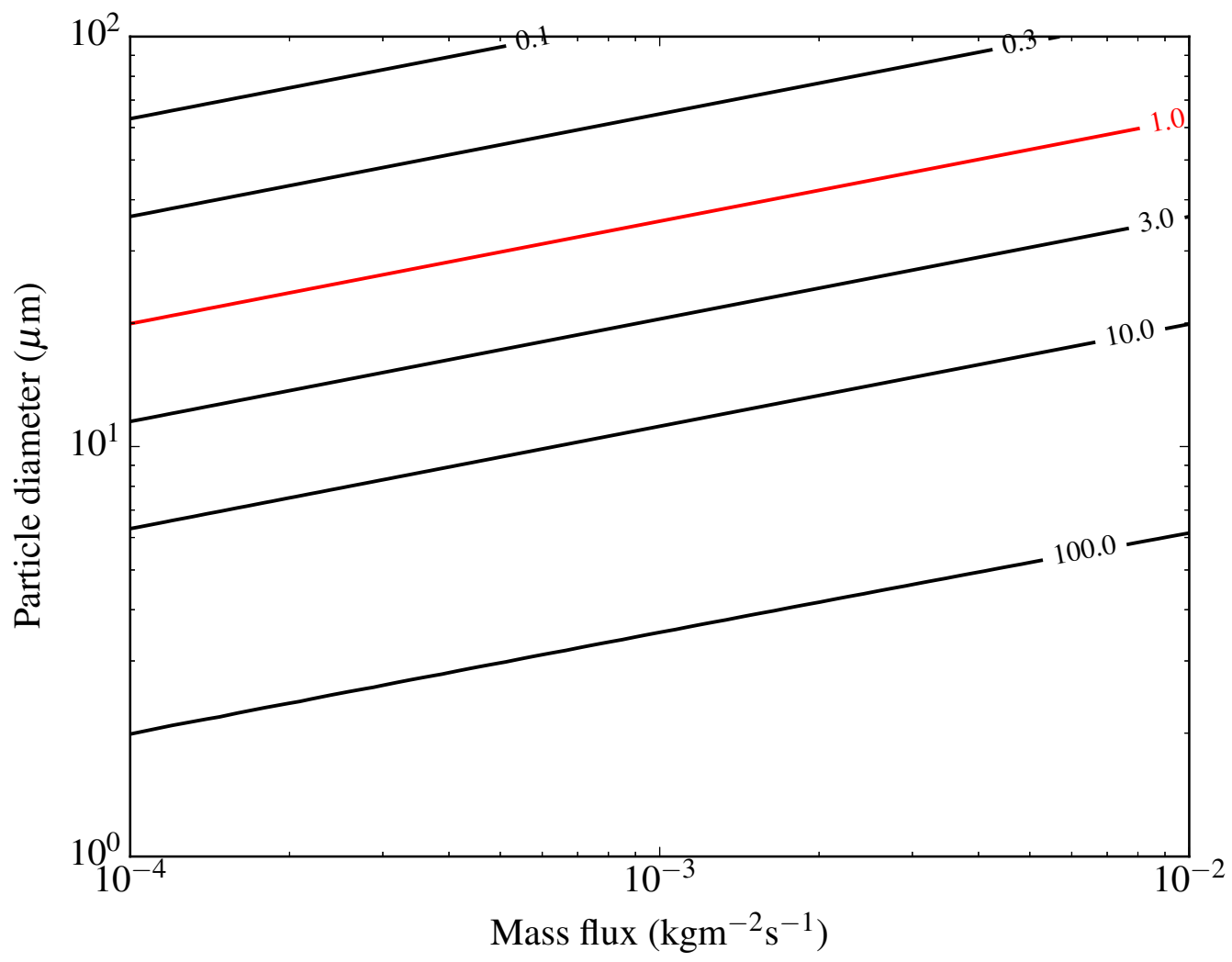

Figure 4: A contour plot of $B_{v i}$, computed using estimates for the particle volume fraction and layer thickness, at $t=10 \mathrm{~s}$ for various mass fluxes and particle diameters. The line $B_{v i}=1$ is highlighted in red. The plot further reinforces the finding that higher mass flux and/or smaller particle diameter encourages plume formation. 
by setting $B$ equal to unity, $h_{\text {crit }}$ was derived. For $B_{v v}$, the value of $h_{\text {crit }}$ is given by

$$
h_{\mathrm{crit}}=\sqrt{\frac{d_{p}^{4} g\left(\rho_{p}-\rho_{f}\right)}{18 \mu_{f} \dot{V}_{p}}} .
$$

On the assumption that $\rho \approx \rho_{f}$ in (7) because $\alpha_{f} \approx 1$, and that plumes have formed when $\delta=h$, the value of $h_{\text {crit }}$ for $B_{v i}$ is given by

$$
h_{\text {crit }}=\left(\frac{2 \rho_{f}}{\beta}\right)\left(\frac{\left(\rho_{p}-\rho_{f}\right)^{2} g^{2} d_{p}^{6}}{5832 \mu_{f}^{3} \dot{V}_{p}}\right) .
$$

Figures $5 \mathrm{a}$ and $5 \mathrm{~b}$ illustrate the relationship between the particle diameter and the critical value $h_{\text {crit }}$ for both measures, for all volumetric flux rates considered in this paper. While all the values of $h_{\text {crit }}$ and $h$ were such that $h_{\text {crit }}<h<H$ was satisfied, a measure could only be considered meaningful and useful if the expected $h_{\text {crit }}$ values consistently agree with the actual values of $h$ at the time of plume formation (i.e. if the values of $h_{\text {crit }}$ run parallel to all the layer thicknesses determined from the numerical simulations). As demontrated in Figure 5a, this is clearly not the case for the $B_{v v}$ measure whose values for $h_{\text {crit }}$ start to diverge from the theoretical prediction. In contrast, the values of $h_{\text {crit }}$ obtained from the $B_{v i}$ measure, which takes into account the inertial drag acting on the particles, run parallel to all the data points as shown in Figure 5b. This further demonstrates the robustness and applicability of the $B_{v i}$ measure when the exact values of $h, \delta$ and $\alpha_{p}$ are not readily available.

Since the volumetric flux and particle diameter are two quantities that are often known during or after an eruption event, a plot of $h_{\text {crit }}$ (for the $B_{v i}$ measure) against the volumetric flux for various particle diameters is given 

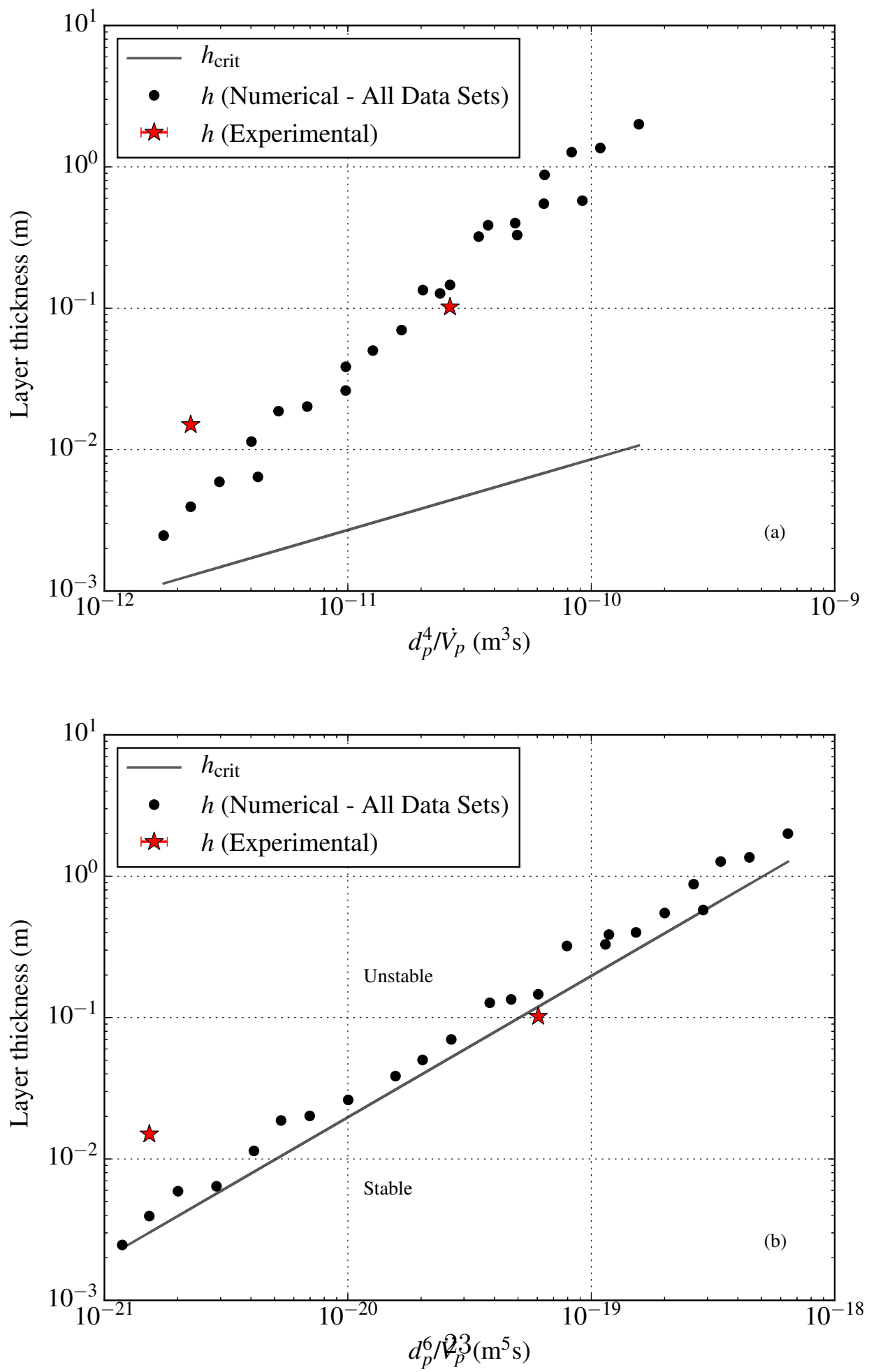

Figure 5: Plots of layer thickness $h$ and the critical layer thickness $h_{\text {crit }}$ for (a) the $B_{v v}$ measure, and (b) the $B_{v i}$ measure. The quantities $d_{p}^{4} / \dot{V}_{p}$ and $d_{p}^{6} / \dot{V}_{p}$ (which are functions of the particle diameter $d_{p}$ and the volumetric flux $\dot{V}_{p}$ ) were considered along the $x$-axis to allow all data points to be plotted against a single $h_{\text {crit }}$ line (rather than having a separate line for each volumetric flux or particle diameter). 
in Figure 6 for reference. This also helps to demonstrate once again how increasing the volumetric flux rate and/or decreasing the particle diameter makes the system increasingly unstable, as shown by the smaller $h_{\text {crit }}$ values.

In the context of subaqueous explosive volcanic eruptions, in which the near-surface layer is formed from particles being forced upwards, high inertia and buoyancy are necessary to sustain particle ascent before the eruption column spreads out laterally along the water's surface (White, 2000; White et al. (2003), pp. 9-12). If the mass flux of particles at the surface is greater than that typically achieved by atmospheric ash fallout, then Figure 6 implies that a much thinner layer will be required to initiate plume onset (for a given particle diameter). It is also important to note that, since plume size is related to $h$ and therefore $h_{\text {crit }}$, any eruption column that is unable to sustain its upward motion and is thicker than $h_{\text {crit }}$ will collapse as a density current/plume, regardless of whether the ash particles reach the water's surface.

\subsection{Including Additional Particle Sizes}

All the simulations presented thus far have considered multiphase flows comprising ash particles of the same diameter, known as monodisperse flows. Such flows are certainly an idealisation since real volcanic ash particles can vary greatly in diameter (Rose and Durant, 2009). The inclusion of additional particle phases each defined by a different particle diameter, forming a so-called polydisperse flow (Crowe et al. (1998), p. 37), can therefore significantly alter the behaviour and enhance the realism of the results. To investigate the effect of multiple particle diameters on the transport of ash in water, and to determine how the theoretical measures defined earlier should 


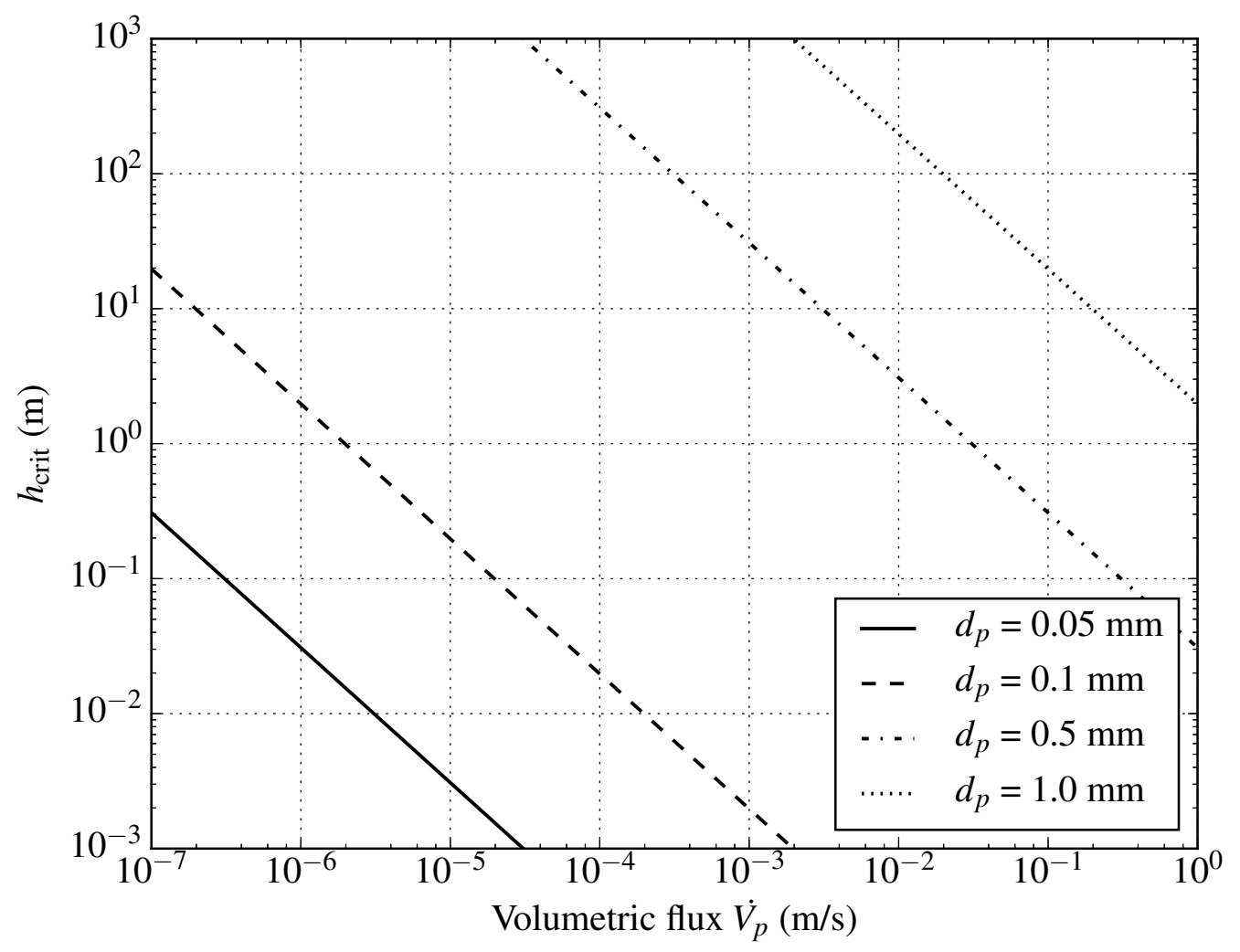

Figure 6: Plot of critical layer thickness $h_{\text {crit }}$ (from the $B_{v i}$ measure) against volumetric flux $\dot{V}_{p}$, for various particle diameters. The volumetric fluxes (per unit area) used in the experiments by Carey (1997) are of $O\left(10^{-7}\right) \mathrm{ms}^{-1}$. 
be modified to support polydisperse flows, a three-phase simulation was set up in Fluidity which extended the earlier two-phase simulations.

Two particle diameters $d_{p_{1}}=26 \mu \mathrm{m}$ and $d_{p_{2}}=48 \mu \mathrm{m}$ in the range of those considered by Carey (1997) were employed. Both particle phases had the same density of $2,340 \mathrm{kgm}^{-3}$. A previously used (total) mass flux of $4.72 \times 10^{-4} \mathrm{kgm}^{-2} \mathrm{~s}^{-1}$ was chosen and remained constant, but was divided equally between the two particle phases such that each one fluxed in at $2.36 \times 10^{-4} \mathrm{kgm}^{-2} \mathrm{~s}^{-1}$. All other aspects of the set-up remained the same as the earlier two-phase simulations.

After performing the simulation, it was found that at early times the 26 $\mu \mathrm{m}$ particles and $48 \mu \mathrm{m}$ particles behaved just like their monodisperse versions. That is, Stokes' law settling ensued once the particles first entered the water tank, as shown by the good agreement with the Stokes' law velocities of $0.00049 \mathrm{~ms}^{-1}$ and $0.00168 \mathrm{~ms}^{-1}$ (for $d_{p_{1}}=26 \mu \mathrm{m}$ and $d_{p_{2}}=48 \mu \mathrm{m}$, respectively) in Figure 7. The near-surface layer of particles that formed was essentially divided up into two parts as a result of the different settling velocities; the smaller $26 \mu \mathrm{m}$ particles formed their own relatively thin and more concentrated 'sub-layer', while the larger $48 \mu \mathrm{m}$ particles were able to overtake the $26 \mu \mathrm{m}$ particles and form a thicker layer as shown in Figures 8a and 8f. After the initial growth of the layer (as a whole), plumes formed from the thinner sub-layer layer of $26 \mu \mathrm{m}$ particles while the layer of $48 \mu \mathrm{m}$ particles remained almost uniform in shape, as shown in Figures 8b and 8g. This occurred at approximately the same time as the monodisperse $26 \mu \mathrm{m}$ simulation, but the plumes grew at a slightly slower rate which may have been the result of the presence of larger particles that typically increase the 
stability of the system. Despite this small difference, the dynamics of the each particle phase were qualitatively similar to the monodisperse simulations of $26 \mu \mathrm{m}$ and $48 \mu \mathrm{m}$ particles up until this point.

The plumes of $26 \mu \mathrm{m}$ particles that grew from the thin sub-layer eventually started to influence the dynamics of the other part of the layer composed solely of $48 \mu \mathrm{m}$ particles, which were still settling at near-Stokes' law velocity, by entraining them. The growth of any small instabilities in the $48 \mu \mathrm{m}$ particle sub-layer was essentially over-ridden by the presence of the plumes of smaller particles. Therefore, while the two particle phases behaved almost independently at early times, in a similar manner to the separate monodisperse versions, it was the smaller particles in the system that influenced the dynamics of the whole polydisperse system at later times.

As the plumes continued to grow and entrain material the two particle phases became strongly coupled to one another (as shown by the similar velocity profiles in Figure 7 at late times). This resulted in their volume fraction fields becoming almost identical in shape (see Figures $8 \mathrm{c}-\mathrm{e}$ and $8 \mathrm{~h}-$ j). The plumes were of a comparable length to those composed solely of $26 \mu \mathrm{m}$ particles, although they appeared to be a few millimetres thicker as a result of the larger particles. Furthermore, as the plumes descended, the smaller particles tended to move a small distance away from the surface of the plumes and instead drift behind a thin outer layer of larger particles because of drag reduction effects. This suggests that a degree of sorting by settling velocity takes place during collective particle descent and deposition, which is commonly seen in the real world (Carey, 1997; Manville and Wilson, 2004). 


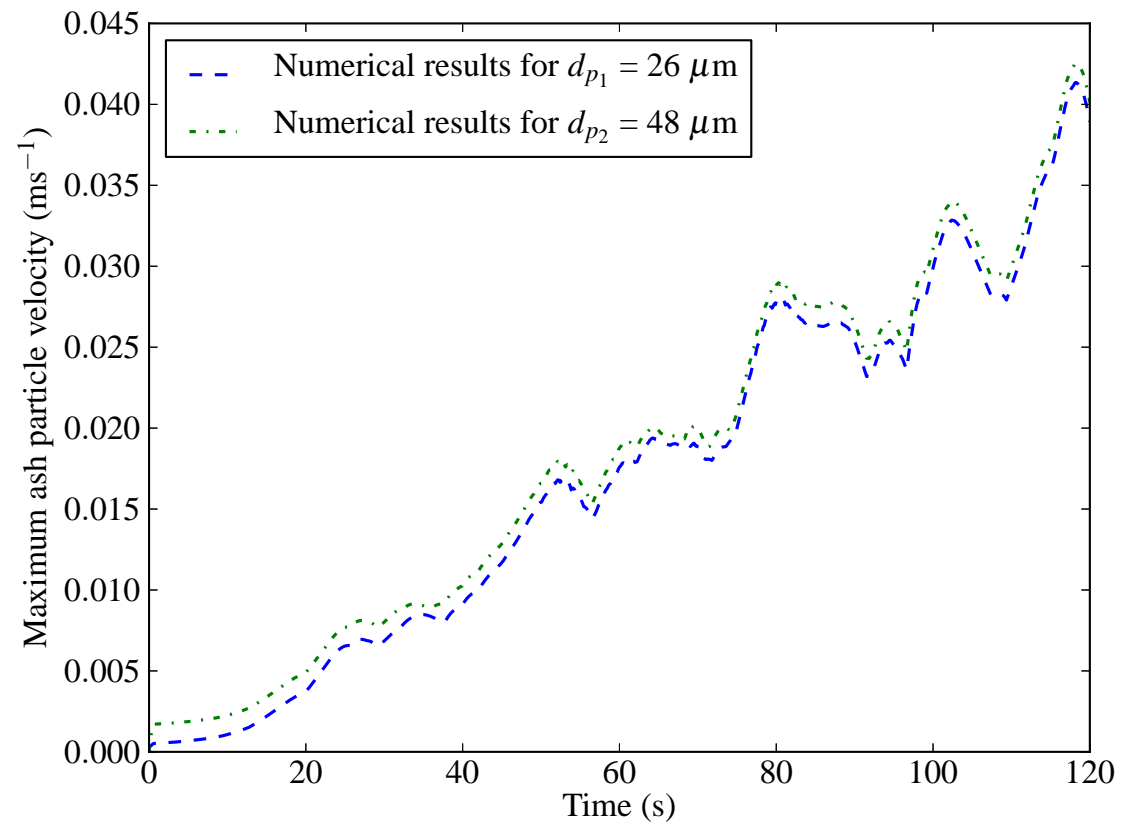

Figure 7: Maximum velocity of ash particles in each particle phase against time, with $d_{p_{1}}=26 \mu \mathrm{m}$ and $d_{p_{2}}=48 \mu \mathrm{m}$, in a two-dimensional polydisperse simulation of the experiments by Carey (1997). 

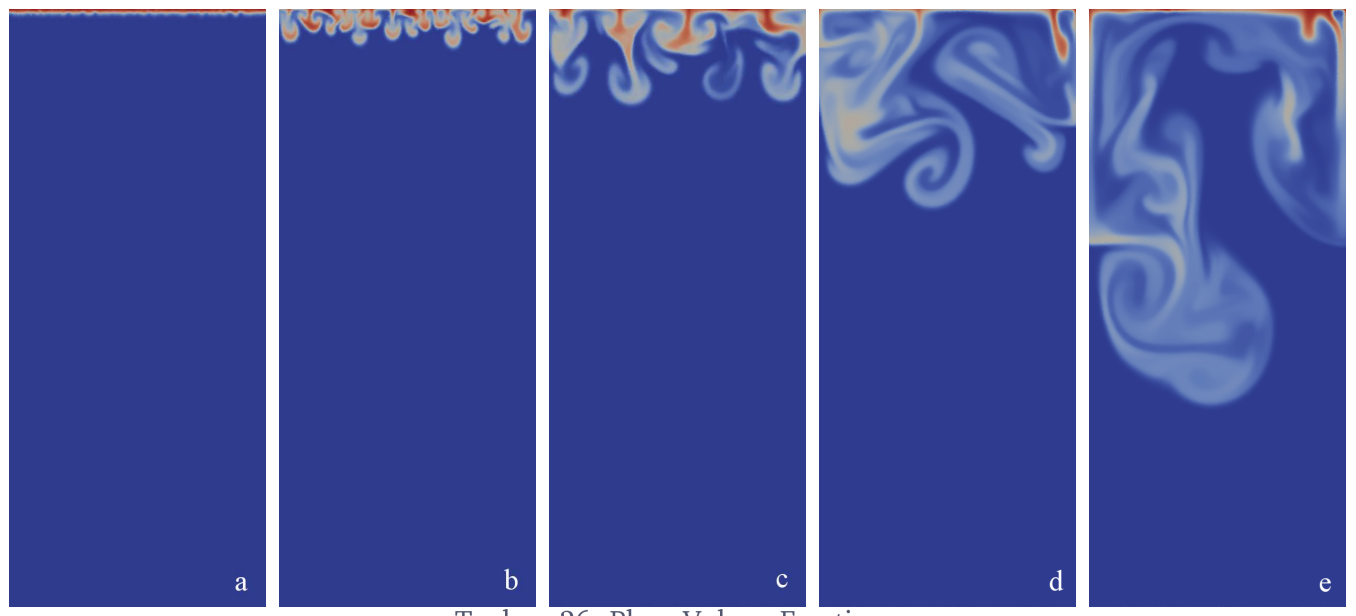

Tephra_26::PhaseVolumeFraction

$\begin{array}{lllll}4 e-5 & 8 e-5 & 0.0001 & 0.0002 & 0.0002\end{array}$

$1 e-7$

0.00021
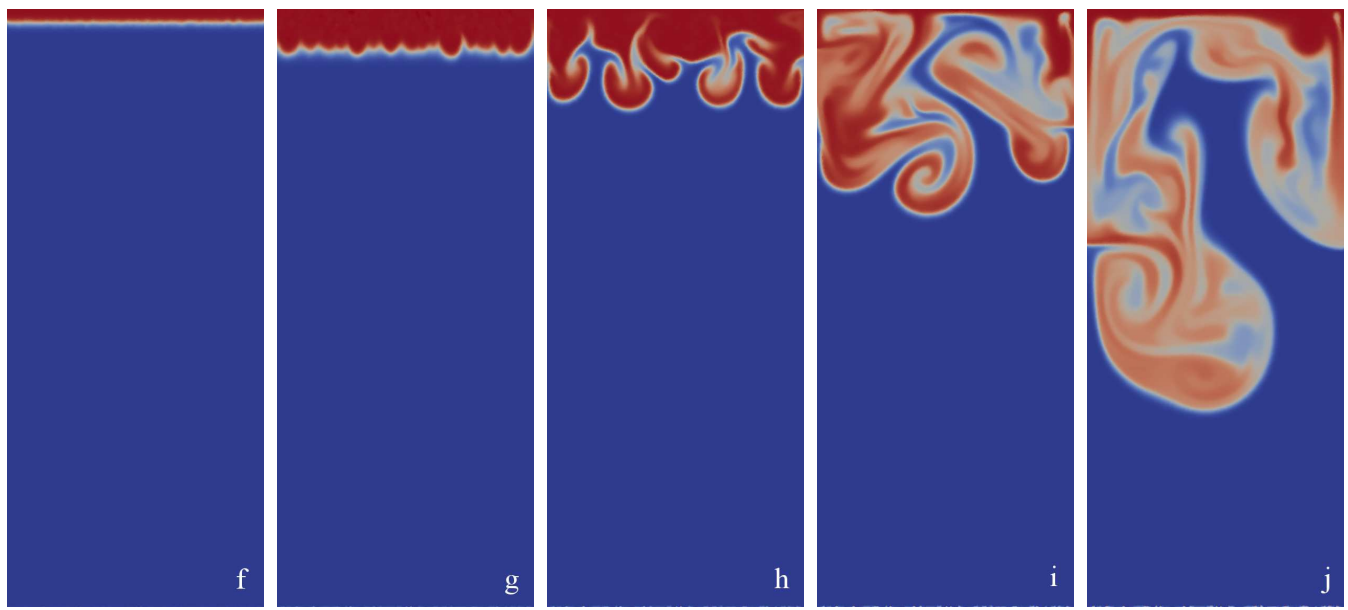

Tephra_48::PhaseVolumeFraction

$2 e-5$

$4 \mathrm{e}-5$

$6 e-5$

$1 \mathrm{e}-7$

6.1e-5

Figure 8: Visualisation of a three-phase, polydisperse ash settling simulation in Fluidity, with $d_{p_{1}}=26 \mu \mathrm{m}$ (top row) and $d_{p_{2}}=48 \mu \mathrm{m}$ (bottom row), at $t=10,30,50,80$ and 120 $\mathrm{s}$ (from left to right). The volume fraction of the particle phase $\left(\alpha_{p}\right)$ is shown; warmer colours represent a higher volume fraction. All visualisations show the whole $0.3 \mathrm{~m} \times 0.7$ m domain. 


\begin{tabular}{|c|c|c|c|}
\hline Reference & Mass flux $\left(\mathrm{kgm}^{-2} \mathrm{~s}^{-1}\right)$ & $d_{p_{1}}(\mu \mathrm{m})$ & $d_{p_{2}}(\mu \mathrm{m})$ \\
\hline P1 & $4.72 \times 10^{-4}$ & 20 & 26 \\
P2 & $4.72 \times 10^{-4}$ & 26 & 32 \\
P3 & $4.72 \times 10^{-4}$ & 32 & 48 \\
P4 & $4.72 \times 10^{-4}$ & 48 & 64 \\
\hline
\end{tabular}

Table 2: Reference table for the polydisperse simulations in the numerical parameter study.

Since the theoretical measures of the tendency for plume formation depend on the particle diameter, it is worth considering how the measures should be modified to support multiple particle diameters. To this end, four additional polydisperse simulations were performed. The particle diameters chosen covered the range used by Carey (1997) and are detailed in Table 2.

For the purpose of computing the dimensionless quantities $B_{v v}$ and $B_{v i}$, plumes were once again said to have formed when $\delta=h$. However, the calculation of the layer thickness through Stokes' law (and also the calculation of $\tau_{\text {individual }}$ ) needs to be considered carefully. It has already been shown here that the dynamics of ash settling in water can be affected heavily by the end members of the particle size range, so simply using an average for $d_{p}$ when computing both the layer thickness and $\tau_{\text {individual }}$ may not be accurate in general. It is also not appropriate to define the layer thickness as the maximum of the thicknesses of the two 'sub-layers' that form within the whole near-surface layer, because the thicker sub-layer (comprising larger particles) will eventually become entrained within the plumes growing from the shallow sub-layer (comprising smaller particles). It is because of this reason that using the Stokes' law settling velocity of the smaller particles 
instead of the larger particles gave a good estimation of the layer thickness. Therefore, when computing $h$ and $\tau_{\text {individual }}, d_{p}$ was chosen to be equal to $d_{p_{1}}$.

The results from the parameter study of the polydisperse simulations are plotted in Figure 9 (for the $B_{v i}$ measure only). Once again, the values for $B_{v v}$ did not lie consistently on a particular contour, whereas the measure that took into account the balance between gravitational forces and inertial drag $\left(B_{v i}\right)$ did. Moreover, this particular contour was approximately the same as the one from the monodisperse simulations, suggesting that the measures are robust even when multiple particle sizes are considered. Note also that only the definition of the layer thickness and $d_{p}$ (in $\left.\tau_{\text {individual }}\right)$ needed to be treated carefully; the formulation of the dimensionless quantity itself did not need to be changed.

\section{Discussion}

By once again assuming that $\rho \approx \rho_{f}$ in (7) because $\alpha_{f} \approx 1$, a useful property of (7) is that $B_{v i}$ is a function of the product of $h$ and $\alpha_{p}$, which is the volume of particles per unit area in the particle-laden water layer at the onset of pluming. Assuming that material reaching the sea or lake floor by plumes spreads laterally as it is deposited to form a semi-continuous layer of approximately uniform thickness, mass conservation implies that the final deposit should contain the same volume of particles per unit area as the original particle-water layer. Hence, (7) provides a measure of the tendency for plumes to form which can be calculated from the properties of the final deposit: the product of the volume fraction of particles in the deposit $\alpha_{p \text {,deposit }}$ and the deposit thickness $h_{\text {deposit }}$. Knowledge of the mass flux and duration 


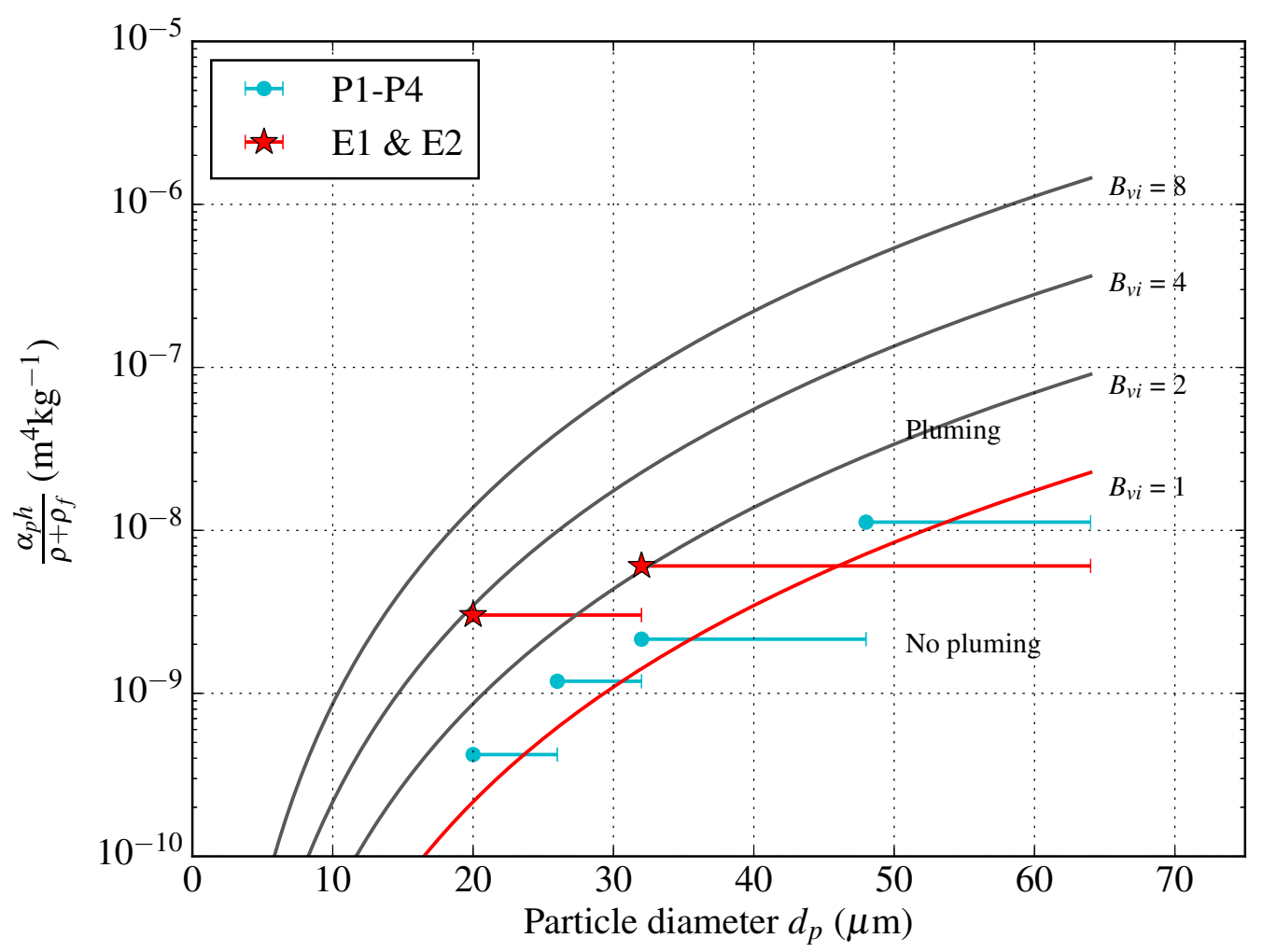

Figure 9: $B_{v i}$ results from the four polydisperse simulations (points $\mathrm{P} 1-\mathrm{P} 4$, detailed in Table 2), using $\delta=h$. Several contours of $B_{v i}$ are given by solid lines. Note that the $x$ coordinate of each numerical data point corresponds to the smallest particle diameter used in each polydisperse simulation, since this value is used to compute $B_{v i}$. As before, points E1 and E2 correspond to the experimental data; however, in light of the findings from the polydisperse simulations, the smallest particle diameters from the original experiments by Carey $(1997)\left(d_{p}=20 \mu \mathrm{m}\right.$ and $\left.d_{p}=32 \mu \mathrm{m}\right)$ were used instead of the averages given in Table 1. 


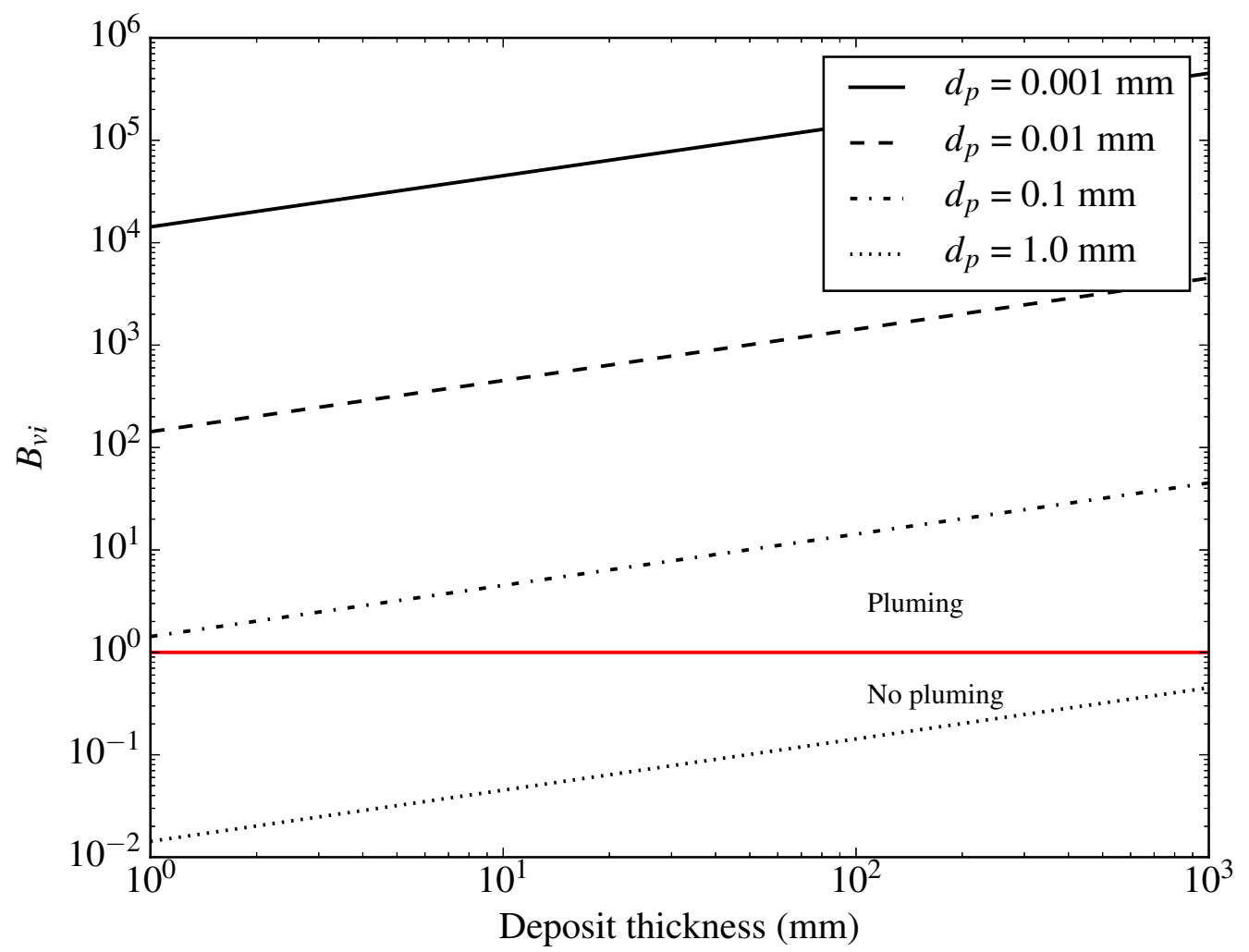

Figure 10: $B_{v i}$ as a function of deposit thickness for various particle diameters. The horizontal red line represents $B_{v i}=1$ and indicates the threshold for stability; values of $B_{v i}>1$ imply that plume formation is likely.

are not required. Adopting this approach, Figure 10 shows how $B_{v i}$ depends on the deposit thickness for various particle diameters. A particle volume fraction of 0.55 has been assumed for the final deposit, based on typical bulk densities of compacted wet ash (Macedonio and Costa, 2012).

The plot shows that for particles smaller than $0.1 \mathrm{~mm}$ in diameter, $B_{v i}$ is greater than unity for final deposits thicker than $1 \mathrm{~mm}$, suggesting that plume formation is expected in the formation of most benthic ash deposits, 
particularly thick (single) deposits or those comprised of fine particles. On the other hand, a larger particle diameter helps to stabilise the system and prevent pluming. For $d_{p} \geq 1 \mathrm{~mm}, B_{v i}$ values are less than unity for the range of deposit thicknesses considered, suggesting that in such cases the Stokes' law settling velocity is high enough to prevent a concentrated particlewater layer from building up near the surface and causing Rayleigh-Taylor instabilities to develop.

The Reynolds number is a useful dimensionless quantity for determining whether viscous or inertial drag effects dominate the dynamics. By defining separate Reynolds numbers for individual particles and plumes, one can decide which $B$ measure is appropriate for a given geophysical scenario. These Reynolds numbers are respectively defined as

$$
\operatorname{Re}_{\text {particle }}=\frac{\rho_{f}\left|\mathbf{u}_{p}\right| d_{p}}{\mu_{f}},
$$

and

$$
\operatorname{Re}_{\text {plume }}=\frac{\rho_{f}\left|\mathbf{u}_{\text {plume }}\right| d_{\text {plume }}}{\mu_{f}},
$$

where $\left|\mathbf{u}_{\text {plume }}\right|$ and $d_{\text {plume }}$ are the velocity and length scale of the plume.

The $B_{v i}$ measure is appropriate in cases where $\mathrm{Re}_{\text {particle }}$ is small and $\mathrm{Re}_{\text {plume }}$ is typically much greater than unity, implying that individual particle and plume settling are dominated by viscous and inertial drag, respectively. For micrometre-sized ash particles settling in water with a velocity that obeys Stokes' law, this is clearly the case for $\operatorname{Re}_{\text {particle }}$ (e.g. $O\left(10^{-2}\right)$ for the $48 \mu \mathrm{m}$ particles considered here). In contrast, $\operatorname{Re}_{\text {plume }} \gg 1$ as the plume diameter and velocity is typically several times (or even several orders of magnitude) larger than those of the individual particles, as shown by the numerical simu- 
lations presented in this paper and the original experiments by Carey (1997). The measure $B_{v i}$ is therefore appropriate in this case.

In addition to ash particles settling through bodies of water, the new measure $B_{v i}$ may also be applicable to other geophysical processes which have the potential to form plumes. One example is the settling of volcanic ash through the atmosphere following an explosive volcanic eruption event. For small pyroclasts, $\mathrm{Re}_{\text {particle }}$ will still be less than unity (typically between $O(1)$ and $O\left(10^{-5}\right)$ for fine ash of the same size and a similar density to that considered here (Bonadonna et al., 1998)) if Stokes' law continues to hold, while the sheer diameter (tens to hundreds of metres) and settling velocity of the growing plumes results in $\mathrm{Re}_{\text {plume }}$ becoming large enough to imply that inertial drag forces dominate the plume's dynamics. However, it is important to note that the individual descent of larger pyroclasts will be controlled by inertial rather than viscous drag as a result of their size. Furthermore, unlike the particles settling in water, individual particles may begin their descent with a high inertia. This is certainly the case for impact ejecta re-entering the atmosphere, for example. In these cases, Stokes' law will no longer hold and the $B_{i i}$ measure given in Appendix B may be more appropriate.

The process of crystals settling in a magma chamber is another example of where a different measure is necessary (Marsh, 1988). Here, the dynamics of the particles will obey Stokes' law regardless of whether they settle individually or collectively due to the high viscosity of the ambient fluid. In this scenario, the $B_{v v}$ measure would be more appropriate. However, unlike water or air, any significant variation in the viscosity of the magma would need to be taken into account. 


\section{Conclusion}

This paper presented a new measure of the tendency for volcanic ash particle plumes to form in water which, unlike existing measures, takes into account the fact that plume growth and descent are controlled by the balance between gravitational forces and inertial (rather than viscous) drag. The measure was evaluated, along with a measure by Marsh (1988) that assumes Stokes' law-based (i.e. viscous drag-controlled) collective settling, using results from a suite of particle settling simulations and previous analogue experiments by Carey (1997). The measure that assumes collective settling is slowed by viscous drag $\left(B_{v v}\right)$ did not consistently predict the onset of pluming and in some cases grossly under-estimated the timescale of collective particle settling. In contrast, the new measure that assumes collective settling is slowed by inertial drag $\left(B_{v i}\right)$ correctly predicted plume onset conditions for all numerical simulations, and was much more consistent with experimental data, highlighting the need to take the inertial drag force into account

The robustness of the new measure became even more apparent when it was re-arranged in terms of a critical layer thickness $h_{\text {crit }}$, such that the layer thickness must satisfy $h_{\text {crit }}<h<H$ (where $H$ is the height of the water column) for pluming to occur. This quantity requires only the volumetric flux of particles and the particle diameter to be known, and is therefore more suitable in field studies. The values of $h_{\text {crit }}$ for the $B_{v v}$ measure did not consistently agree with the layer thicknesses determined from the numerical simulations, and in fact diverged away from them. This means that $B_{v v}$ cannot be used to robustly predict the tendency for plumes to form, since the 
corresponding values of $h_{\text {crit }}$ imply that plumes may form much sooner than they actually do. In contrast, the layer thicknesses all ran parallel to the $h_{\text {crit }}$ line for the new measure as expected, further reinforcing its validity. The ability of the new measure to predict plume onset accurately and consistently allows the residence times and deposition rates of particles in a large body of water to be determined more reliably. The measure therefore has significant implications for geological field studies since it permits the improved interpretation of the layers of volcaniclastic material along the seabed.

The formulation of the new measure itself brought an additional benefit; the value of $B_{v i}$ could be estimated from the properties of the final deposit, such that knowledge of the particle mass flux and duration are not required. It was found that for typical fine-grained ash deposits greater than $1 \mathrm{~mm}$ in thickness, it is likely that particles would have settled collectively as plumes. However, care must be taken when using this estimation since it introduces assumptions about the layer itself (e.g. uniform in thickness) which may not always be justifiable in practice.

Despite the study focussing mainly on monodisperse systems with just one particle size, it was demonstrated that the measure can also correctly predict plume onset conditions for a polydisperse flow. Plume onset was found to be governed by the smaller particles in such flows, so the value of $d_{p}$ in $B_{v i}$ should be chosen to be the diameter of the smallest particle in the system. Furthermore, it is worth noting that while the new measure was only applied to situations involving volcanic ash, it is likely that it will also be valid for other geophysical events involving small particles in water, such as impact ejecta fallout. 


\section{Acknowledgments}

CTJ and SCK were funded by the Institute of Shock Physics at Imperial College London and the Atomic Weapons Establishment; GSC was funded by the Natural Environment Research Council, Fellowship Grant NE/E013589/1. Support from the Imperial College High Performance Computing Service was also gratefully received. The interested reader is referred to the thesis by Jacobs (2013), from which most of the content in this paper is based. The authors would like to thank Vernon Manville and an anonymous reviewer for their constructive feedback which greatly improved the quality of this paper. AWE (C) Crown Owned Copyright (2012).

\section{Appendix A. Notation}

A list of notation used throughout this paper is given in Table A.3.

\section{Appendix B. Derivation of the $B_{i i}$ measure}

At high Reynolds numbers the terminal velocity of an individual particle can be approximated by balancing the inertial drag force with the buoyancy force and the particle's weight:

$$
\frac{1}{2} C_{\mathrm{D}} A_{p} \rho_{f}|\mathbf{u}|^{2}=\frac{1}{6}\left(\rho_{p}-\rho_{f}\right) g \pi d_{p}^{3}
$$

where $C_{\mathrm{D}}$ and $A_{p}$ are the drag coefficient and cross-sectional area of a spherical particle, respectively (Batchelor (1973), pp. 233-234). Using the expression $A_{p}=\frac{1}{4} \pi d_{p}^{2}$ and re-arranging for the particle speed $|\mathbf{u}|$ gives

$$
|\mathbf{u}|=\sqrt{\frac{4\left(\rho_{p}-\rho_{f}\right) g d_{p}}{3 C_{\mathrm{D}} \rho_{f}}},
$$




\begin{tabular}{|c|c|c|}
\hline Notation & Units & Description \\
\hline$t$ & $\mathrm{~s}$ & Time \\
\hline$t_{\text {onset }}$ & $\mathrm{s}$ & Time of plume onset \\
\hline$\tau_{\text {individual }}$ & s & Timescale of individual particle settling \\
\hline$\tau_{\text {collective }}$ & $\mathrm{s}$ & Timescale of collective particle settling \\
\hline$\alpha_{p}$ & Dimensionless & Volume fraction of the particles \\
\hline$\alpha_{f}$ & Dimensionless & Volume fraction of the fluid \\
\hline$\rho_{p}$ & $\operatorname{kgm}^{-3}$ & Density of the particles \\
\hline$\rho_{f}$ & $\mathrm{kgm}^{-3}$ & Density of the fluid \\
\hline$\rho$ & $\mathrm{kgm}^{-3}$ & Bulk density $\left(\rho=\alpha_{f} \rho_{f}+\alpha_{p} \rho_{p}\right)$ \\
\hline $\mathbf{u}_{p}$ & $\mathrm{~ms}^{-1}$ & Velocity of the particles \\
\hline $\mathbf{u}_{f}$ & $\mathrm{~ms}^{-1}$ & Velocity of the fluid \\
\hline$\mu_{f}$ & Pa s & Viscosity of the fluid \\
\hline$g$ & $\mathrm{~ms}^{-2}$ & Acceleration due to gravity \\
\hline$d_{p}$ & $\mathrm{~m}$ & Diameter of the particles \\
\hline $\operatorname{Re}$ & Dimensionless & Reynolds number \\
\hline At & Dimensionless & Atwood number \\
\hline$h$ & $\mathrm{~m}$ & Thickness of the near-surface layer \\
\hline$h_{\text {crit }}$ & $\mathrm{m}$ & Critical layer thickness \\
\hline$H$ & $\mathrm{~m}$ & Height of the water column \\
\hline$\delta$ & $\mathrm{m}$ & Maximum amplitude of the growing plumes \\
\hline$B_{v v}$ & Dimensionless & The measure by Marsh (1988) \\
\hline$B_{v i}$ & Dimensionless & The new measure presented in this paper \\
\hline$\beta$ & Dimensionless & Constant plume growth parameter \\
\hline$\dot{M}_{p}$ & $\mathrm{kgm}^{-2} \mathrm{~s}^{-1}$ & Mass flux (per unit area) of particles \\
\hline$\dot{V}_{p}$ & $\mathrm{~ms}^{-1}$ & Volumetric flux (per unit area) of particles \\
\hline$A$ & $\mathrm{~m}^{2}$ & Area through which particles enter the water \\
\hline$V_{p}$ & $\mathrm{~m}^{3}$ & Volume of the near-surface layer occupied by particles \\
\hline$V_{\text {layer }}$ & $\mathrm{m}^{3}$ & Total volume of the near-surface layer (including the water) \\
\hline
\end{tabular}

Table A.3: The notation used throughout this paper. 
and simplifying produces the non-dimensional number $B_{i i}$ :

$$
B_{i i}=\frac{h}{2} \sqrt{\frac{3 C_{\mathrm{D}} \alpha_{p}}{4 \delta d_{p}}} .
$$

Finally, dividing (B.3) by the timescale for inertial drag-based collective

$$
\tau_{\text {collective }}=2 \sqrt{\frac{\rho_{f} \delta}{\left(\rho_{p}-\rho_{f}\right) \alpha_{p} g}},
$$

\section{References}

Batchelor, G. K., 1973. An Introduction to Fluid Dynamics. Cambridge University Press, Cambridge, UK, 615 pages.

Bergantz, G. W., Ni, J., 1999. A numerical study of sedimentation by dripping instabilities in viscous fluids. International Journal of Multiphase Flow 25 (2), 307-320.

Bonadonna, C., Ernst, G. G. J., Sparks, R. S. J., 1998. Thickness variations and volume estimates of tephra fall deposits: the importance of particle Reynolds number. Journal of Volcanology and Geothermal Research 81 (34), 173-187. 
Bramlette, M. N., Bradley, W. H., 1941. Geology and biology of North Atlantic deep-sea cores between Newfoundland and Ireland: lithology and geological interpretation. U.S. Geological Survey Professional Paper 196A, 1 .

Carazzo, G., Jellinek, A. M., 2012. A new view of the dynamics, stability and longevity of volcanic clouds. Earth and Planetary Science Letters 325-326, $39-51$.

Carey, S., 1997. Influence of convective sedimentation on the formation of widespread tephra fall layers in the deep sea. Geology 25 (9), 839-842.

Carey, S., Schneider, J.-L., 2011. Volcaniclastic Processes and Deposits in the Deep-Sea. In: Hüneke, H., Mulder, T. (Eds.), Deep-Sea Sediments. Vol. 63 of Developments in Sedimentology. Elsevier, Ch. 7, pp. 457-515.

Crowe, C. T., Sommerfeld, M., Tsuji, Y., 1998. Multiphase Flows with Droplets and Particles. CRC Press, Boca Raton, USA, 471 pages.

Dalziel, S. B., Patterson, M. D., Caulfield, C. P., Coomaraswamy, I. A., 2008. A numerical study of sedimentation by dripping instabilities in viscous fluids. Physics of Fluids 20 (6), 065106.

Davies, D. R., Wilson, C. R., Kramer, S. C., 2011. Fluidity: A fully unstructured anisotropic adaptive mesh computational modeling framework for geodynamics. Geochemistry Geophysics Geosystems 12 (6).

Dimonte, G., Schneider, M., 2000. Density ratio dependence of RayleighTaylor mixing for sustained and impulsive acceleration histories. Physics of Fluids 12 (2), 304-321. 
Dimonte, G., Youngs, D. L., Dimits, A., S, W., Marinak, M., Wunsch, S., C., G., Robinson, A., Andrews, M. J., Ramaprabhu, P., Calder, A. C., Fryxell, B., Biello, J., Dursi, L., MacNeice, P., Olson, K., Ricker, P., Rosner, R., Timmes, F., Tufo, H., Young, Y.-N., Zingale, M., 2004. A comparative study of the turbulent Rayleigh-Taylor instability using high-resolution three-dimensional numerical simulations: The Alpha-Group collaboration. Physics of Fluids 16 (5), 1668-1693.

Geuzaine, C., Remacle, J.-F., 2009. Gmsh: A 3-D finite element mesh generator with built-in pre- and post-processing facilities. International Journal for Numerical Methods in Engineering 79 (11), 1309-1331.

Goldin, T., 2008. Atmospheric Interactions During Global Deposition of Chicxulub Impact Ejecta. Ph.D. thesis, University of Arizona.

Jacobs, C. T., 2013. Modelling of Multiphase Flows on Adaptive Unstructured Meshes with Applications to the Dynamics of Volcanic Ash Plumes. Ph.D. thesis, Imperial College London.

Jacobs, C. T., Collins, G. S., Piggott, M. D., Kramer, S. C., Wilson, C. R. G., 2013. Multiphase flow modelling of volcanic ash particle settling in water using adaptive unstructured meshes. Geophysical Journal International 192 (2), 647-665.

Kuenen, P. H., 1968. Settling convection and grain-size analysis. Journal of Sedimentary Research 38 (3), 817-831.

Ledbetter, M. T., Sparks, R. S. J., 1979. Duration of large-magnitude explo- 
sive eruptions deduced from graded bedding in deep-sea ash layers. Geology 7 (5), 240-244.

Macedonio, G., Costa, A., 2012. Brief Communication "Rain effect on the load of tephra deposits". Natural Hazards and Earth System Sciences $12(4), 1229-1233$.

Manville, V., Wilson, C., 2004. Vertical density currents: a review of their potential role in the deposition and interpretation of deep-sea ash layers. Journal of the Geological Society, London 161, 947-958.

Marsh, B., 1988. Crystal Capture, Sorting, and Retention in Convecting Magma. Geological Society of America Bulletin 100, 1720-1737.

Narbonne, G. M., 2005. The Ediacara Biota: Neoproterozoic Origin of Animals and Their Ecosystems. Annual Review of Earth and Planetary Sciences 33, 421-442.

Piggott, M. D., Gorman, G. J., Pain, C. C., Allison, P. A., Candy, A. S., Martin, B. T., Wells, M. R., 2008. A new computational framework for multi-scale ocean modelling based on adapting unstructured meshes. International Journal for Numerical Methods in Fluids 56 (8), 1003-1015.

Ristorcelli, J. R., Clark, T. T., 2004. Rayleigh-Taylor turbulence: self-similar analysis and direct numerical simulations. Journal of Fluid Mechanics 507, $213-253$.

Rose, W. I., Durant, A. J., 2009. Fine ash content of explosive eruptions. Journal of Volcanology and Geothermal Research 186 (1-2), 32-39. 
Sarna-Wojcicki, A., Shipley, S., Waitt, R., Dzurisin, D., Wood, S., 1981. Areal distribution, thickness, mass, volume, and grain size of air-fall ash from the six major eruptions of 1980. In: Lipman, P., Mullineaux, D. (Eds.), The 1980 Eruptions of Mount St. Helens, Washington. U.S. Geological Survey Professional Paper 1250. pp. 577-600.

Scheidegger, K., Federman, A., Tallman, A., 1982. Compositional heterogeneity of tephras from the 1980 eruptions of Mount St. Helens. Journal of Geophysical Research 87 (B13), 10,861-10,881.

Seilacher, A., Reif, W.-E., Westphal, F., 1985. Sedimentological, Ecological and Temporal Patterns of Fossil Lagerstätten. Philosophical Transactions of the Royal Society of London. Series B, Biological Sciences 311 (1148), $5-23$.

Stokes, G. G., 1851. On the Effect of the Internal Friction of Fluids on the Motion of Pendulums. Transactions of the Cambridge Philosophical Society 9,8 .

Ver Straeten, C. A., 2004. K-bentonites, volcanic ash preservation, and implications for Early to Middle Devonian volcanism in the Acadian orogen, eastern North America. Geological Society of America Bulletin 116 (3-4), $474-489$

Ver Straeten, C. A., 2008. Volcanic Tephra Bed Formation and Condensation Processes: A Review and Examination from Devonian Stratigraphic Sequences. The Journal of Geology 116 (6), 545-557. 
White, J. D. L., 2000. Subaqueous eruption-fed density currents and their deposits. Precambrian Research 101 (2-4), 87-109.

White, J. D. L., Smellie, J. L., Clague, D. A., 2003. Explosive Subaqueous Volcanism. American Geophysical Union, Washington, DC, USA, 379 pages.

Whitehead, J., Luther, D., 1975. Dynamics of Laboratory Diapir and Plume Models. Journal of Geophysical Research 80 (5), 705-717.

Wiesner, M., Wang, Y., Zheng, L., 1995. Fallout of volcanic ash to the deep South China Sea induced by the 1991 eruption of Mount Pinatubo (Philippines). Geology 23 (10), 885-888.

Youngs, D. L., 1984. Numerical simulation of turbulent mixing by RayleighTaylor instability. Physica D: Nonlinear Phenomena 12 (1-3), 32-44. 